Original Research Paper

\title{
The Dual Exponentiated Weibull Model
}

\author{
Mona Mustafa Elbiely \\ Department of Statistics, Mathematics and Insurance, Damanhur University, Egypt
}

Article history

Received: 07-04-2019

Revised: 24-05-2019

Accepted: 29-06-2019

Email: elbielym@gmail.com

\begin{abstract}
A new lifetime model with various shapes of the hazard rate function for modeling uni-modal and bimodal data sets is introduced and studied along with its statistical properties. Before using the maximum likelihood method for estimating the unknown model parameters, we assessed its performance via a simulation study. The flexibility of the new model is illustrated via plots of the probability and hazard rate functions for three real data applications.
\end{abstract}

Keywords: Exponentiated Weibull, Order Statistics, Quantile Function, Moments, Moments Generating Function, Maximum Likelihood Estimation, Simulation

\section{Introduction}

Many extensions of the Weibull (W) distribution are presented in the literature. Among these models, the Exponentiated Weibull (EW) is the most popular one (Mudholkar et al., 1993). A Random Variable (RV) $T$ is said to have the EW distribution if its Probability Density Function (PDF) and Cumulative Distribution Function (CDF) are given by:

$$
\pi_{\alpha, \beta}(t)=\alpha \beta t^{\beta-1} \exp \left(-t^{\beta}\right)\left[1-\exp \left(-t^{\beta}\right)\right]^{\alpha-1}
$$

and:

$$
\prod_{\alpha, \beta}(t)=\left[1-\exp \left(-t^{\beta}\right)\right]^{a},
$$

respectively, for $t>0, \alpha>0$, and $\beta>0$. For $\alpha=1$, we have the standard one parameter Weibull model (Weibull (1951). For $\beta=1$; we have the one parameter exponentiated exponential model (Gupta and Kundu (1999). For $\beta=2$, we have the standard one parameter Burr type $X$ distribution. Cordeiro et al. (2017) suggested a new flexible family called the Exponentiated Weibull-H (EW-H) family, the CDF of the EW-H family is given by:

$$
F_{\theta, \lambda}(x)=\left\{1-\exp \left[-\left(\frac{\Pi(x)}{\bar{\Pi}(x)}\right)^{\lambda}\right]\right\}^{\theta},
$$

where, $\lambda>0$ and $\theta>0$ are two shape parameters, $\Pi(x)$ is the CDF of the base line model. The PDF of the EW-H class corresponding to (3) reduces to:

$$
\begin{aligned}
& f_{\theta, \lambda}(x)=\lambda \theta \frac{\bar{\Pi}(x)^{-\lambda-1}}{\Pi(x)^{-\lambda+1}} \exp \left[-\left(\frac{\Pi(x)}{\bar{\Pi}(x)}\right)^{\lambda}\right] \\
& \times \pi(x)\left\{1-\exp \left[-\left(\frac{\Pi(x)}{\bar{\Pi}(x)}\right)^{\lambda}\right]\right\}^{\theta-1},
\end{aligned}
$$

where, $\pi(x)=\frac{d}{d x} \Pi(x)$ is the PDF of the base line model and $\bar{\Pi}(x)=1-\Pi(x)$. Hassan and Elgarhy (2016) introduced a similar work to Cordeiro et al. (2017), but we depended on Cordeiro et al. (2017) in this work. Using (3) and (2), we construct a model called the Dual Exponentiated Weibull (DEW) with CDF given by:

$$
F_{\theta, \lambda, \alpha, \beta}(x)=\left[1-\exp \left(-\left\{\frac{\left[1-\exp \left(-x^{\beta}\right)\right]^{\alpha}}{1-\left[1-\exp \left(-x^{\beta}\right)\right]^{\alpha}}\right\}^{\lambda}\right)\right]^{\theta}
$$

The PDF of the DEW corresponding to (5) reduces to:

$$
\begin{aligned}
& f_{\theta, \lambda, \alpha, \beta}(x)=\lambda \theta \alpha \beta x^{\beta-1} \exp \left(-x^{\beta}\right) \frac{\left[1-\exp \left(-x^{\beta}\right)\right]^{\alpha \lambda-1}}{\left\{1-\left[1-\exp \left(-x^{\beta}\right)\right]^{\alpha}\right\}^{\lambda+1}} \\
& \times \exp \left(-\left\{\frac{1-\exp \left(-x^{\beta}\right)^{\alpha}}{1-\left[1-\exp \left(-x^{\beta}\right)\right]^{\alpha}}\right]^{\lambda}\right) \\
& \times\left[1-\exp \left(-\left\{\frac{\left[1-\exp \left(-x^{\beta}\right)\right]^{\alpha}}{1-\left[1-\exp \left(-x^{\beta}\right)\right]^{\alpha}}\right\}\right)\right]^{\theta-1} .
\end{aligned}
$$


A Random Variable (RV) $X$ having PDF $f_{\theta, \lambda, \alpha} \beta(x)(6)$ is denoted by $X \sim \operatorname{DEW}(\theta, \lambda, \alpha, \beta)$. The Hazard Rate Function (HRF) can be derived from the well known relationship. The PDF and HRF plots of the DEW model are given in Fig. 1 and 2 respectively. The new additional parameters $\theta$ and $\lambda$ will enable us to study the tail behavior of the new density (6) with more flexibility.
Some cases of the DEW model are presented in Table 1. In Table 1, the H-model refers to the model with CDF:

$$
M(x)=1-\exp [-\Pi(x) / \bar{\Pi}(x)]
$$

which is an exponential model of the odds ratio of a continuous $\mathrm{RV}$ whose $\mathrm{CDF}$ is given by $\Pi(x)$.

Table 1: Some sub models from the DEW model

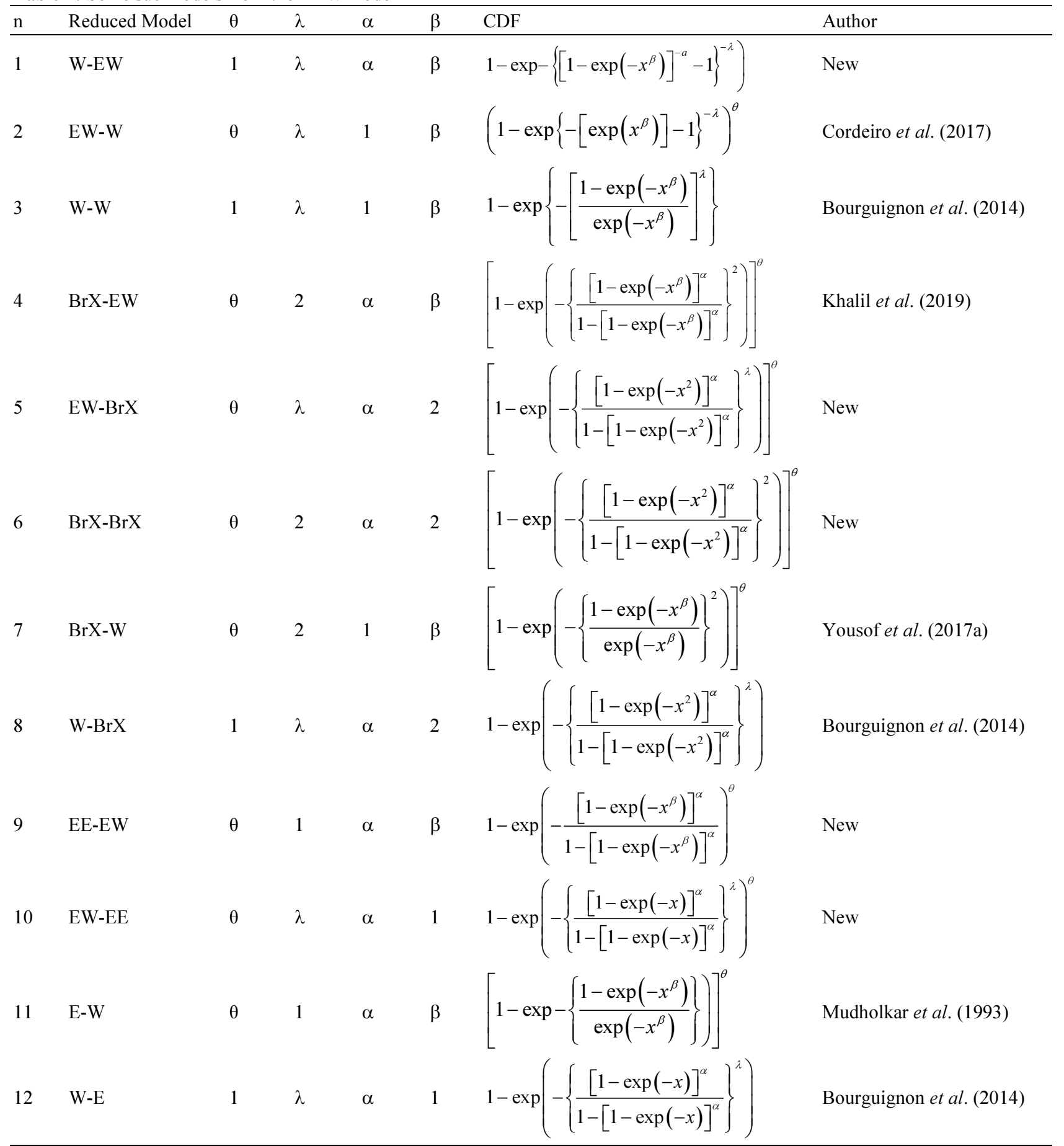


Table 1: Continue

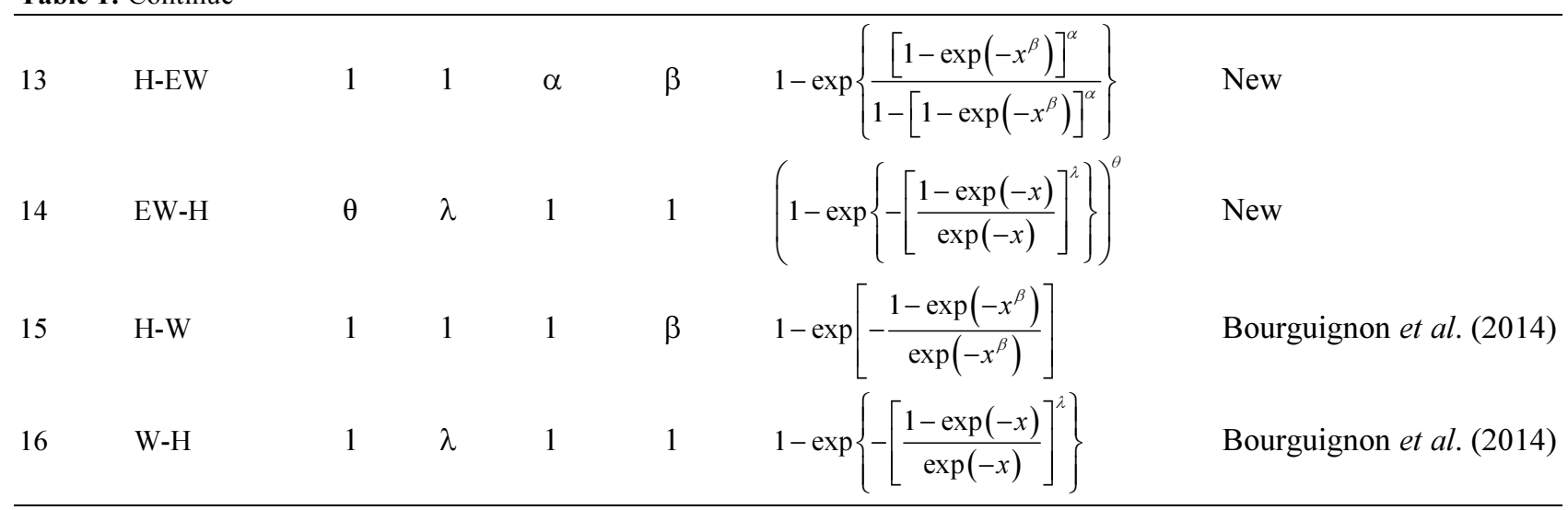

\section{Linear Representation}

A simple linear representation for the DEW density will be provided in this section. If $|q|<1$ and $\alpha>0$ is a real non-integer, the power series holds:

$$
(1-q)^{\alpha-1}=\sum_{m=0}^{\infty} \frac{(-q)^{m} \Gamma(\alpha)}{m ! \Gamma(\alpha-m)} .
$$

Applying (7) to the last term in (6) gives:

$$
\begin{aligned}
& f_{\theta, \lambda, \alpha, \beta}(x)=\lambda \theta \alpha \beta x^{\beta-1} \\
& \times \frac{\left[1-\exp \left(-x^{\beta}\right)\right]^{\alpha \lambda-1}}{\left\{1-\left[1-\exp \left(-x^{\beta}\right)\right]^{\alpha}\right\}^{\lambda+1}} \\
& \times \sum_{i=0}^{\infty} \frac{(-1)^{i} \Gamma(\theta)}{i ! \Gamma(-i+\theta)} \exp \left(-x^{\beta}\right) \\
& \times \exp \left(-(1+i)\left\{\frac{\left[1-\exp \left(-x^{\beta}\right)\right]^{\alpha}}{1-\left[1-\exp \left(-x^{\beta}\right)\right]^{\alpha}}\right\}^{\lambda}\right)
\end{aligned}
$$

Expanding $A_{i}$ in power series, we have:

$$
A_{i}=\sum_{\zeta=0}^{\infty} \frac{(-1)^{\zeta}(i+1)^{\zeta}}{\zeta !} \frac{\left[1-\exp \left(-x^{\beta}\right)\right]^{\zeta \alpha \lambda}}{\left\{1-\left[1-\exp \left(-x^{\beta}\right)\right]^{\alpha}\right\}^{\zeta \lambda}}
$$

Inserting $A_{i}$ in (8), the DEW density reduces to:

$$
\begin{aligned}
f_{\theta, \lambda, \alpha, \beta}(x)= & \alpha \beta x^{\beta-1} \exp \left(-x^{\beta}\right)\left[1-\exp \left(-x^{\beta}\right)\right]^{\alpha \lambda(\zeta+1)-1} \\
& \times \sum_{i, \zeta=0}^{\infty} \frac{(-1)^{\zeta+i} \theta \lambda \Gamma(\theta)(i+1)^{\zeta}}{i ! \zeta ! \Gamma(-i+\theta)}\left\{1-\left[1-\exp \left(-x^{\beta}\right)\right]^{\alpha}\right\}^{-1[1+(\zeta+1) \lambda]},
\end{aligned}
$$

Using the generalized binomial expansion:

$$
(1-q)^{-\alpha}=\left.\sum_{m=0}^{\infty} \frac{\Gamma(a+m)}{m ! \Gamma(a)} q^{m}\right|_{(|q|<1 \text { and } a>0)}
$$

to:

$$
\left\{-\left[1-\exp \left(-x^{\beta}\right)\right]^{\alpha}+1\right\}^{-[1+(\zeta+1) \lambda]}
$$

we can write:

$$
\begin{aligned}
& \left\{-\left[1-\exp \left(-x^{\beta}\right)\right]^{\alpha}\right\}^{-[1+(\zeta+1) \lambda]} \\
& =\sum_{j=0}^{\infty} \frac{\Gamma(\lambda[\zeta+1]+j+1)}{j ! \Gamma(1+\lambda(\zeta+1))}\left[1-\exp \left(-x^{\beta}\right)\right]^{\alpha j} .
\end{aligned}
$$

Inserting (10) in (9), the DEW density can be expressed as an infinite linear combination of EW density function:

$f_{\theta, \lambda, \alpha, \beta}(x)=\sum_{\zeta, j=0}^{\infty} \tau_{\zeta, j} \pi_{[(\zeta+1) \lambda+j] \alpha, \beta}(x)$,

where, $\pi_{[(\zeta+1) \lambda+j] \alpha, \beta}(x)$ is the EW with power parameter $[(\zeta+1) \lambda+j] \alpha$ and:

$$
\tau_{\zeta, j}=\theta \lambda \sum_{i=0}^{\infty} \frac{(-1)^{\zeta+i}(i+1)^{\zeta} \Gamma(\lambda[\zeta+1]+j+1) \Gamma(\theta)}{i ! \zeta ! j\{[(\zeta+1) \lambda+j] \alpha\} \Gamma(-i+\theta) \Gamma(1+\lambda(\zeta+1))} .
$$

Equation (11) reveals that the new PDF of $X$ can be expressed as a linear combination of EW densities. So, several mathematical properties of the new model can be attained by knowing those of the EW distribution. Likewise, the CDF of the DEW density can also be written as:

$$
F_{\theta, \lambda, \alpha, \beta}(x)=\sum_{\zeta, j=0}^{\infty} \tau_{\zeta, j} \Pi_{[(\zeta+1) \lambda+j] \alpha, \beta(x),}
$$

where, $\Pi_{[(\zeta+1) \lambda+j] \alpha, \beta(x)}$ is the CDF of the EW with power parameter $[(\zeta+1) \lambda+j] \alpha$. 

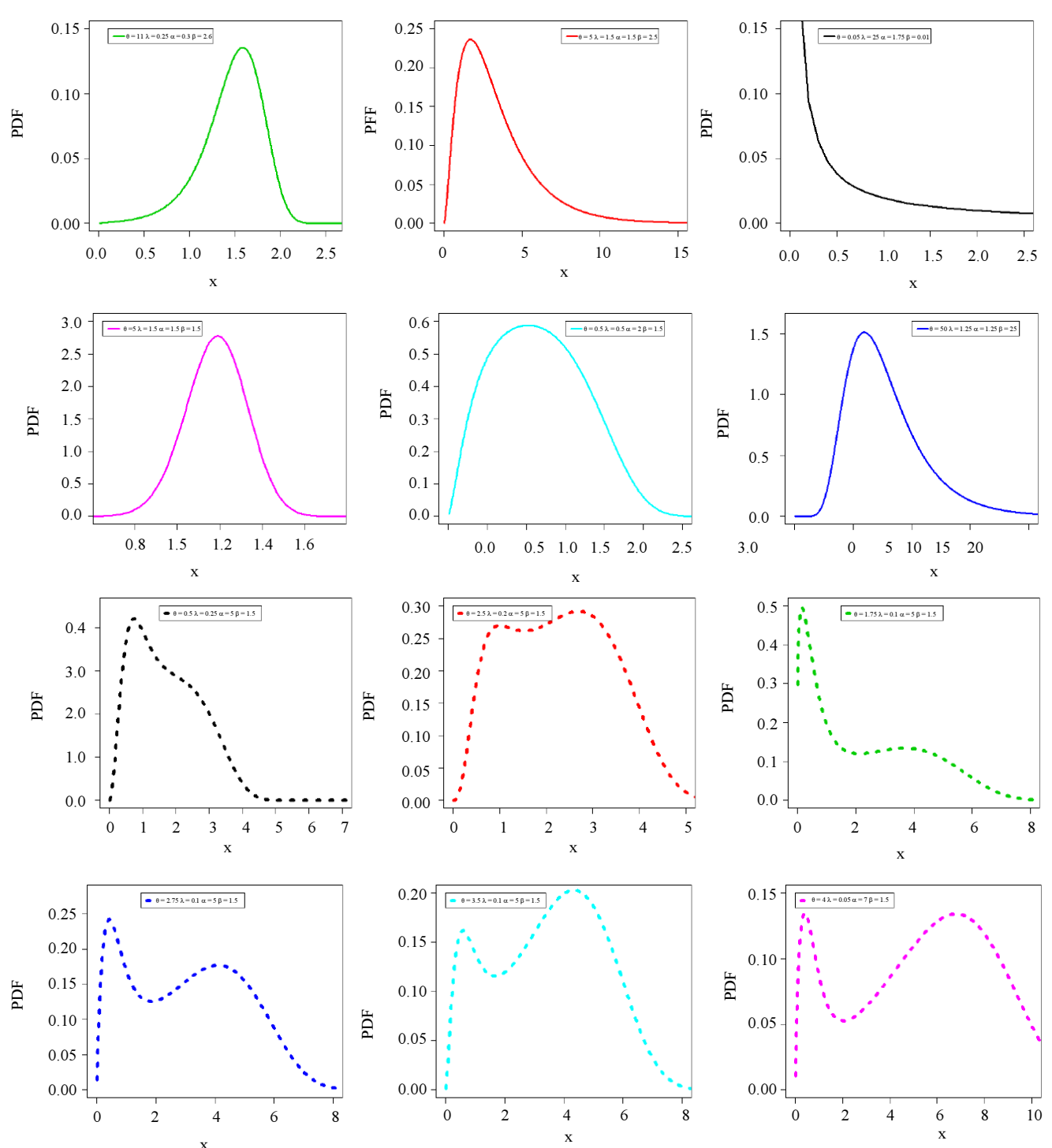

Fig. 1: Plots of the DEW PDF for selected parameter values

\section{Mathematical Properties}

\section{Justification and Graphical Presentation}

We are motivated to introduce the DEW for the following reasons:

1. The DEW model is very suitable to define special models with different kinds of hazard rates. Fig. 1 displays some plots of the PDF of the DEW for different values of $\theta, \lambda, \alpha$ and $\beta$. These plots show that the DEW density can be symmetric or rightskewed or left-skewed or inverted $J$ shape or unimodal or bimodal

2. The HRF plots of the DEW model are given in Fig. 2. The HRF of the DEW model can be increasing or increasing constant or decreasing or upside-down or bathtub, upside-down bathtub or constant or $J$ shaped
3. The DEW model provides the best fit in modeling different types of data sets

\section{Moments}

The $r^{\text {th }}$ moment of $X$, say $\mu_{r}^{\prime}$, follows from Equation (11) as:

$$
\mu_{r}^{\prime}=\boldsymbol{E}\left(X^{r}\right)=\left.\Gamma\left(r \beta^{-1}+1\right) \sum_{\zeta, j, h=0}^{\infty} c_{\zeta, j, h}^{\{r,[(\zeta+1) \lambda+j] \alpha\}}\right|_{(-\beta<r)}
$$

where, $c_{\zeta, j, h}^{\{r,[(\zeta+1) \lambda+j] \alpha\}}=\tau_{\zeta, j} c_{h}^{\{\tau,[(\zeta+1) \lambda+j] \alpha\}}$ and:

$$
c_{h}^{\{r[(\zeta+1) \lambda+j] \alpha\}}=\frac{(-1)^{h}[(\zeta+1) \lambda+j] \alpha}{(h+1)^{\left(r \beta^{-1}+1\right)}}\left(\begin{array}{c}
{[(\zeta+1) \lambda+j] \alpha-1} \\
h
\end{array}\right),
$$


when $r=1$ in (12), we get the mean $(\boldsymbol{E}(X))$ of the new model:

$$
\boldsymbol{E}(X)=\left.\Gamma\left(\beta^{-1}+1\right) \sum_{\zeta, j, h=0}^{\infty} c_{\zeta, j, h}^{\{1,[(\zeta+1) \lambda+j] \alpha\}}\right|_{(-\beta<1)}
$$

Some numerical results for the $\boldsymbol{E}(X)$ and other related measures are given in Table 2.

\section{Quantile Function and Moment Generating Function}

The Quantile Function $(Q F)$ of $X$ is defined by reversing (5). We have:

$Q(u)=F^{-1}(u)=\left[-\operatorname{In}-\left\{\left[-\log \left(-u^{\frac{1}{\theta}}+1\right)\right]^{-\frac{1}{\lambda}}+1\right\}^{\frac{1}{\alpha}}+1\right]^{\frac{1}{\beta}}$

, $0<u<1$.

Next, we provide a formula for the Moment Generating Function; $M_{X}(t)$ of $\mathrm{X}$ can follow from Equation (11) as:

$$
M_{x}(t)=\left.\Gamma\left(r \beta^{-1}+1\right) \sum_{\zeta, j, r, h=0}^{\infty} \boldsymbol{c}_{\zeta, j, h}^{\{r,[(\zeta+1) \lambda+j] \alpha\}}\right|_{(-\beta<r)},
$$

\section{Incomplete Moments}

The $s^{\text {th }}$ incomplete moment, say $\boldsymbol{I}_{s}(t)$, of $X$ can be derived from (11) as:

$$
\begin{aligned}
& \boldsymbol{I}_{s}(t)=\int_{-\infty}^{t} x^{s} f(x) d x=\Upsilon\left(s \beta^{-1}+1,\left(\frac{1}{t}\right)^{\beta}\right) \\
& \left.\sum_{\zeta, j, h=0}^{\infty} c_{\zeta, j, h}^{\{s,[(\zeta+1) \lambda+j] \alpha\}}\right|_{(-\beta<s)},
\end{aligned}
$$

where, $\Upsilon(\tau, v)$ is the incomplete gamma function:

$$
\Upsilon(\tau, v)=\int_{0}^{v} t^{\tau-1} \exp (-t) d t=\sum_{h=0}^{\infty} \frac{(-1)^{h}}{h !(\tau+h)} v^{\tau+h} .
$$

The first incomplete moment $I_{1}(t)$ is given by (14) with $s=1$ as:

$$
\left.\boldsymbol{I}_{1}(t)=\Upsilon 1+\beta^{-1},\left(\frac{1}{t}\right)^{\beta}\right) \sum_{\zeta, j, h=0}^{\infty} \boldsymbol{c}_{\zeta, j, h}^{\{1,[(\zeta+1) \lambda+j] \alpha\}}
$$

\section{Order Statistics}

Let $X_{1}, \ldots, X_{n}$ be a Random Sample (RS) from the DEW distribution. The PDF of the $i^{t h}$ order statistic, say $X_{i: n}$, can be stated as:

$$
f_{i: n}(x)=f_{\theta, \lambda, \alpha, \beta}(x) \sum_{j=0}^{n-1} \frac{(-1)^{j}}{B(i, n+1-i)}\left(\begin{array}{c}
n-i \\
j
\end{array}\right)\left[F_{\theta, \lambda, \alpha, \beta}(x)\right]^{j+i-1},
$$

where, $B(.,$.$) is the beta function. Based on Equations (5)$ and (6), we have:

$$
f_{\theta, \lambda, \alpha, \beta}(x)\left[F_{\theta, \lambda, \alpha, \beta}(x)\right]^{j+i-1}=\sum_{\zeta, m=0}^{\infty} t_{\zeta, m}^{(j+i-1)} \pi_{[(\zeta+1) \lambda+m] \alpha, \beta}(x),
$$

where:

$$
\begin{aligned}
& t_{\zeta, m}^{(j+i-1)}= \\
& \theta \lambda \sum_{l=0}^{\infty} \frac{(-1)^{l+\zeta}(1+l)^{\zeta} \Gamma(\lambda(\zeta+1)+1+m) \Gamma(j \theta+i \theta)}{l ! m ! \Gamma(\lambda(\zeta+1)+1) \Gamma(-l+j \theta+i \theta)\{[(\zeta+1) \lambda+m] \alpha\}} .
\end{aligned}
$$

Substituting (16) in Equation (15), the PDF of $X_{i: n}$ can be expressed as:

$$
f_{i: n}(x)=\sum_{\zeta, m=0}^{\infty} \tau_{\zeta, m} \pi_{[(\zeta+1) \lambda+m] \alpha, \beta}(x),
$$

where, $\pi_{[(\zeta+1) \lambda+m] \alpha, \beta}(x)$ is the EW density with power parameter $[(\zeta+1) \lambda+m] \alpha$ and:

$$
\tau_{\zeta, m}=\sum_{j=0}^{n-i} \frac{(-1)^{j} t_{\zeta, m}^{(j+i-1)}}{B(i, n+1-i)}\left(\begin{array}{c}
n-i \\
j
\end{array}\right) .
$$

The PDF of the DEW order statistics is expressed as a linear combination of EW PDF. The moments of $X_{i: n}$ are given by:

$$
\boldsymbol{E}\left(X_{i: n}^{s}\right)=\left.\Gamma\left(s^{\beta-1}+1\right) \sum_{\zeta, m, h=0}^{\infty} \frac{\tau_{\zeta, m} \boldsymbol{c}_{h}^{\{s,[(\zeta+1) \lambda+j] \alpha\}}}{B(i, n+1-i)}\right|_{(-\beta<s)} .
$$

\section{Probability Weighted Moments (PWMs)}

The $(s, r)^{t h}$ PWM of the DEW distribution, say $\mu_{s ; r}$, can be defined by:

$$
\mu_{s, r}=\boldsymbol{E}\left[F(X)^{r} X^{s}\right]=\int_{-\infty}^{\infty} F(X)^{r} f(x) x^{s} d x
$$

From Equations (3) and (4), we can write:

$$
F(X)^{r} f(x)=\sum_{\zeta, j=0}^{\infty} c_{\zeta, j}^{(r)} \pi_{[(\zeta+1) \lambda+j] \alpha, \beta}(x),
$$

where:

$$
\begin{aligned}
& c_{\zeta, j}^{(r)}= \\
& \theta \lambda \sum_{i=0}^{\infty} \frac{(-1)^{\zeta+i}(i+1)^{\zeta} \Gamma(\theta[r+1]) \Gamma(1+\lambda[\zeta+1]+j)}{i ! j ! \Gamma(\lambda(\zeta+1)+1) \Gamma(r-i+\theta+1)\{[(\zeta+1) \lambda+j] \alpha\}} .
\end{aligned}
$$

Then, $\mu_{s, r}$ can be expressed as: 


$$
\mu_{s, r}=\sum_{\zeta, j=0}^{\infty} c_{\zeta, j}^{(r)} \int_{-\infty}^{\infty} x^{s} \pi_{[(\zeta+1) \lambda+j] \alpha, \beta}(x) d x .
$$

Finally, the $(s, r)^{\text {th }}$ PWM of $\mathrm{X}$ can be given by:

$$
\mu_{s, r}=\left.\Gamma\left(s \beta^{-1}+1\right) \sum_{\zeta, j=0}^{\infty} d_{\zeta, j}^{(r)} c_{h}^{\{s,[(\zeta+1) \lambda+j] \alpha\}}\right|_{(-\beta<s)} .
$$

\section{Numerical Analysis Results for Some Measures}

Numerical analysis results for the $E(X)$, $\operatorname{variance}(X)$, skewness $(X)$ and $\operatorname{kurtosis}(X)$ using (11) and well-known relationships for some selected values of parameter $\theta, \lambda, \alpha$ and $\beta$ are reported in Table 2 . Based on Table 2 we note that:

1. The skewness of the DEW distribution is always positive and can range in the interval $(1.72 ; 93.4)$

2. The kurtosis of the DEW distribution is ranging from 7.06 to 18175.5

3. The mean of the DEW distribution increases as $\theta$ and $\alpha$ increases

4. The mean of the DEW distribution decreases as $\lambda$ increases

Table 2: $\mathrm{E}(\mathrm{X})$, variance $(\mathrm{X})$, skewness $(\mathrm{X})$ and kurtosis $(\mathrm{X})$ of the DEW distribution

\begin{tabular}{llllrrrr}
\hline$\theta$ & $\lambda$ & $\alpha$ & $\beta$ & $\mathrm{E}(\mathrm{X})$ & variance $(\mathrm{X})$ & \multicolumn{1}{c}{ skewness $(\mathrm{X})$} & kurtosis $(\mathrm{X})$ \\
\hline 0.5 & 0.50 & 0.10 & 0.2 & 0.03402 & 0.78193 & 93.36140 & 18175.4800 \\
1 & & & & 0.06789 & 1.56121 & 66.10060 & 9111.3260 \\
5 & & & 0.33414 & 7.70358 & 29.85600 & 1859.4610 \\
10 & & & 0.65586 & 15.16610 & 21.36170 & 952.3557 \\
50 & & & 2.90117 & 68.28620 & 10.31830 & 223.1026 \\
100 & & & 5.18343 & 123.70200 & 7.83282 & 129.2139 \\
1000 & & & & 622.90300 & 3.93418 & 34.20156 \\
3 & 0.50 & 0.25 & 0.25 & 1.20275 & 24.64500 & 10.78990 & 204.6200 \\
& 1.50 & & & 0.00194 & $4.03 \times \mathrm{e}^{-5}$ & 10.57980 & 222.7260 \\
& 1.75 & & & 0.00094 & $7.48 \times \mathrm{e}^{-6}$ & 9.16693 & 167.6580 \\
2 & 0.50 & 0.20 & 0.25 & 0.50430 & 8.30000 & 15.27990 & 413.5810 \\
& & 0.75 & & 6.22640 & 347.00000 & 6.95833 & 81.4296 \\
& 2.00 & & 26.27700 & 3019.00000 & 4.56574 & 35.3904 \\
& & 10.00 & & 162.69000 & 4680.00000 & 2.75827 & 14.2361 \\
& & 50.00 & & 634.69000 & 3573.00000 & 1.93860 & 8.28824 \\
& 100.00 & & 1031.44000 & 73571.00000 & 1.71939 & 7.06335 \\
0.5 & 0.50 & 0.15 & 0.05110 & 0.38800 & 47.25020 & 4943.2100 \\
& 1.00 & & 0.20 & 0.044700 & 0.11020 & 22.21120 & 933.9750 \\
& & & 0.25 & 0.046700 & 0.06310 & 13.74480 & 326.3040 \\
& & & 0.35 & 0.057610 & 0.04270 & 7.60136 & 91.1281 \\
& & & 0.40 & 0.064600 & 0.04050 & 6.21374 & 59.6693 \\
& & & 0.5 & 0.07970 & 0.04030 & 4.57647 & 31.9450 \\
\hline
\end{tabular}
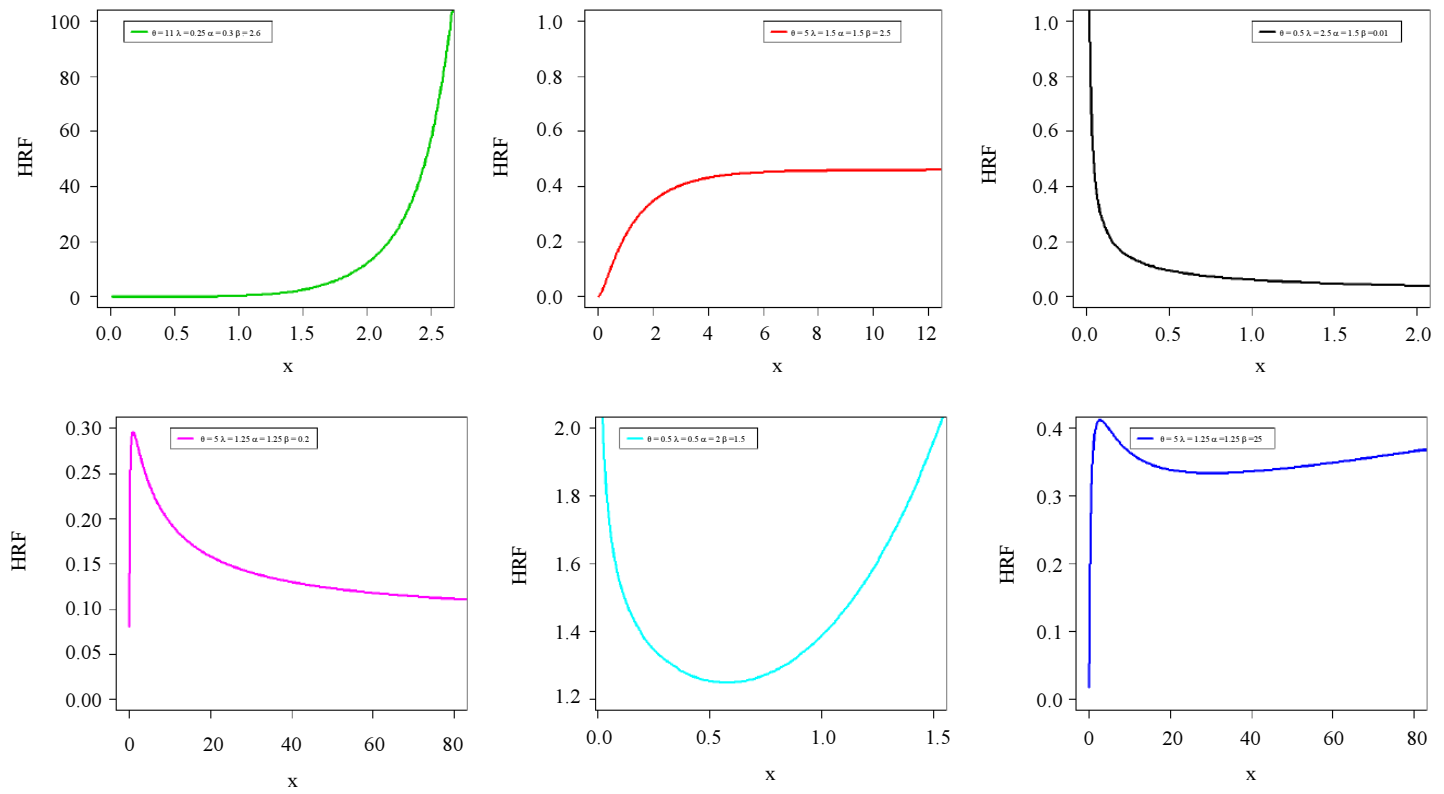

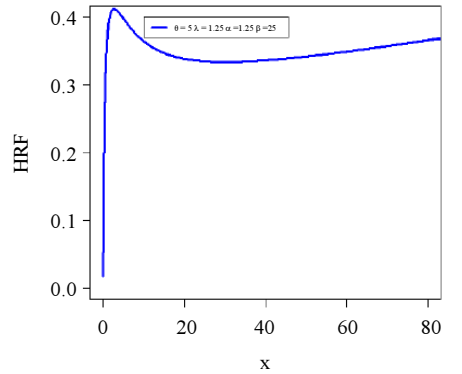



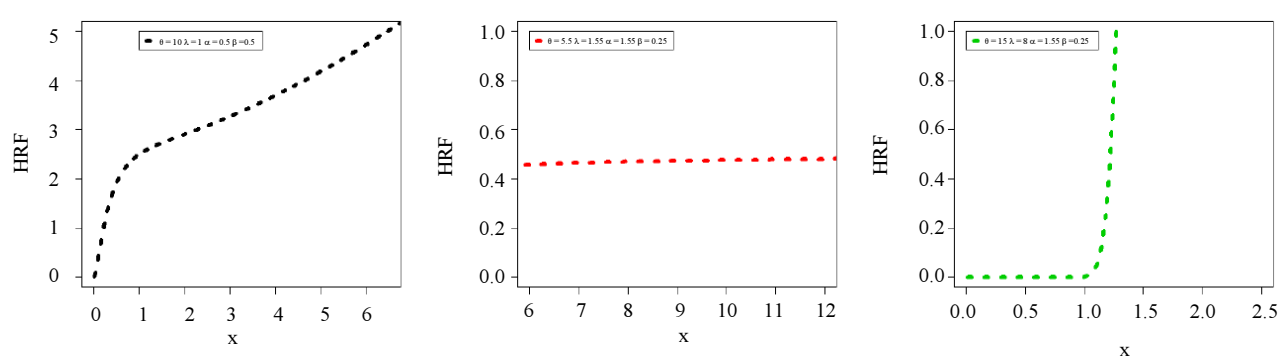

Fig. 2: Plots of the DEW HRF for selected parameter values

\section{Estimation}

Let $x_{1}, \ldots, x_{\mathrm{n}}$ be a RS from the DEW model with parameters $\theta, \lambda, \alpha$ and $\beta$ and let $\Upsilon=(\theta, \lambda, \alpha, \beta)^{\mathrm{T}}$ be $(4 \times 1)$ parameter vector. The log-likelihood function $\ell$ for the DEW distribution is given by:

$$
\begin{aligned}
\ell= & n(\log \theta+\log \lambda+\log \alpha+\log \beta) \\
& -\sum_{i=1}^{n} p_{i}^{\lambda}+(\alpha \lambda-1) \sum_{i=1}^{n} \log s_{i}-\sum_{i=1}^{n} x_{i}^{\beta} \\
& +(\beta-1) \sum_{i=1}^{n} x_{i}+(\theta-1) \sum_{i=1}^{n} \log q_{i} \\
& -(\lambda+1) \sum_{i=1}^{n} \log \left(1-s_{i}^{a}\right),
\end{aligned}
$$

and

where, $s_{i}=1-\exp \left(-x_{i}^{\beta}\right), p_{i}=s_{i}^{\alpha} /\left(1-s_{i}^{\alpha}\right)$

$q_{i}=1-\exp \left(-p_{i}^{\lambda}\right) \mid(i=1, \ldots, n)$.

\section{Simulation Results}

Using (13), we simulate the DEW model via taking $n$ $=20,50,200,500$ and 1000 following 3 sets of parameter values

$$
\begin{array}{ccccc} 
& \theta & \lambda & \alpha & \beta \\
\text { I : } & 3.75 & 2.50 & 4.25 & 0.25 \\
\text { II: } & 2.50 & 1.25 & 2.25 & 0.50 \\
\text { III : } & 0.50 & 0.75 & 2.50 & 0.50
\end{array}
$$

We evaluate the ML Estimations (MLEs) of the parameters for each sample size. Then, by repeating this process $N=1000$ times and calculate the Mean Squared Errors (MSEs) and the Averages of the Estimates (AEs). The numerical results in Table 3 show that both MSEs of $\hat{\theta}, \hat{\lambda}, \hat{\alpha}$ and $\hat{\beta}$ decline toward 0 once $n$ increases for all initial values of $\theta, \lambda, \alpha$ and $\beta$. As $n$ increases, MSEs tend to 0 and the estimates appear to approach the true parameter values confirming the consistency property of the method of maximum likelihood estimation. Table 3 below presents the AEs, MSEs based on $N=$ 1000 simulations of the DEW model for certain values $\theta, \lambda$, $\alpha$ and $\beta$.

\section{Applications}

We present three applications to display the potentiality of the new PDF. To study the difference between the fits of the DEW model and other EW distributions, we will use the statistics of AndersonDarling $\left(\mathrm{A}^{\star}\right)$ and Cramér-von Mises $\left(\mathrm{W}^{\star}\right)$. The MLE and the its Standard Errors (SEs) of the DEW model parameters are specified in Tables 4, 6 and 8. The numerical values of the $A^{\star}$ and $W^{\star}$ are listed in Tables 5, 7 and 9. The estimated CDF, estimated PDF, P-P plot, estimated HRF, Total Time on Test (TTT) plot and Kaplan-Meier survival plot of the three data sets of the DEW model are showed in Fig. 3, 4 and 5. We will specify some competitive models in the following three subsections, however many useful extensions of $\mathrm{W}$ model are available a potential competitive models, these models are presented by Nadarajah et al. (2013); Brito et al. (2017); Hamedani et al. (2017); Alizadeh et al. (2017); Merovci et al. (2017); Yousof et al. (2017b; 2018); Hamedani et al. (2018); Mahmoudi et al. (2018); Cordeiro et al. (2018); Korkmaz et al. (2019), among others. For more details about data set I (failure times of 84 aircraft windshield), II (remission times of cancer patients) and III (strengths of glass fibers); Lee and Wang (2003); Smith and Naylor (1987) respectively.

\section{Modeling Failure Times}

The data consist of 84 observations of failure times. Here, we assess the differences between the fits of the DEW distribution with those of other competitive models, namely: Burr-Hatke EW (BHEW), Ku- maraswamy transmuted W (KwTW), Marshall Olkin extended W (MOEW), Gamma W (GaW), W Fréchet (WFr), Poisson Topp Leone-W (PTLW), transmuted exponentiated generalized W (TEGW), Kumaraswamy W (KwW), Beta W (BW), Transmuted modified W (TMW), Modified beta W (MBW), Mcdonald W (McW), distributions, whose PDFs for $x>0$. The parameters of the above PDFs are all positive real numbers except for the TExGW and TMW models. Table 4 list the values of MLEs and SE for all fitted models. Table 5 reveals that the DEW distribution gives the best fit to failure times data set. 
Mona Mustafa Elbiely / Journal of Mathematics and Statistics 2019, Volume 15: 122.135 DOI: $10.3844 / j m s s p .2019 .122 .135$

Table 3: The AEs and MSEs bsed on $N=1000$ simulations

\begin{tabular}{|c|c|c|c|c|c|c|c|c|c|}
\hline \multirow[b]{2}{*}{$\mathrm{n}$} & $\Upsilon$ & $\mathrm{AE}$ & MSE & $\Upsilon$ & $\mathrm{AE}$ & MSE & $\Upsilon$ & $\mathrm{AE}$ & MSE \\
\hline & \multicolumn{3}{|l|}{$\mathrm{I}$} & \multicolumn{3}{|l|}{ II } & \multicolumn{3}{|c|}{ III } \\
\hline \multirow[t]{4}{*}{20} & $\theta$ & 3.7657 & 0.7120 & $\theta$ & 2.4927 & 1.26480 & $\theta$ & 0.7112 & 1.1011 \\
\hline & $\lambda$ & 2.4985 & 0.0213 & $\lambda$ & 1.2578 & 0.11280 & $\lambda$ & 0.8224 & 0.5233 \\
\hline & $\alpha$ & 4.2709 & 0.0455 & $\alpha$ & 2.2275 & 0.1318 & $\alpha$ & 2.7377 & 1.1261 \\
\hline & $\beta$ & 0.4032 & 0.3126 & $\beta$ & 0.6853 & 0.3644 & $\beta$ & 0.7278 & 0.4838 \\
\hline \multirow[t]{4}{*}{50} & $\theta$ & 3.7327 & 0.2513 & $\theta$ & 2.5313 & 0.44860 & $\theta$ & 0.6623 & 1.0187 \\
\hline & $\lambda$ & 2.4973 & 0.0079 & $\lambda$ & 1.2515 & 0.01180 & $\lambda$ & 0.7901 & 0.4101 \\
\hline & $\alpha$ & 4.2515 & 0.0160 & $\alpha$ & 2.2273 & 0.01370 & $\alpha$ & 2.6925 & 0.9601 \\
\hline & $\beta$ & 0.3539 & 0.2023 & $\beta$ & 0.6566 & 0.23230 & $\beta$ & 0.6511 & 0.2973 \\
\hline \multirow[t]{4}{*}{200} & $\theta$ & 3.7319 & 0.0865 & $\theta$ & 2.4973 & 0.14920 & $\theta$ & 0.5623 & 0.5701 \\
\hline & $\lambda$ & 2.4918 & 0.0027 & $\lambda$ & 1.2297 & 0.00150 & $\lambda$ & 0.7823 & 0.3009 \\
\hline & $\alpha$ & 4.2292 & 0.0056 & $\alpha$ & 2.2283 & 0.00490 & $\alpha$ & 2.6021 & 0.4445 \\
\hline & $\beta$ & 0.3145 & 0.1701 & $\beta$ & 0.6138 & 0.12650 & $\beta$ & 0.6030 & 0.1015 \\
\hline \multirow[t]{4}{*}{500} & $\theta$ & 3.7398 & 0.0255 & $\theta$ & 2.4991 & 0.04851 & $\theta$ & 0.5178 & 0.1799 \\
\hline & $\lambda$ & 2.4905 & 0.0008 & $\lambda$ & 0.2292 & 0.00010 & $\lambda$ & 0.7577 & 0.1010 \\
\hline & $\alpha$ & 4.2296 & 0.0016 & $\alpha$ & 1.2284 & 0.00160 & $\alpha$ & 2.5201 & 0.1329 \\
\hline & $\beta$ & 0.2622 & 0.0492 & $\beta$ & 0.5321 & 0.05611 & $\beta$ & 0.5430 & 0.0522 \\
\hline \multirow[t]{4}{*}{1000} & $\theta$ & 3.7295 & 0.0122 & $\theta$ & 2.4969 & 0.02234 & $\theta$ & 0.4901 & 0.0022 \\
\hline & $\lambda$ & 2.4901 & 0.0002 & $\lambda$ & 1.2298 & 0.00005 & $\lambda$ & 0.7292 & 0.0041 \\
\hline & $\alpha$ & 4.2292 & 0.0008 & $\alpha$ & 2.2296 & 0.00091 & $\alpha$ & 2.5021 & 0.0025 \\
\hline & $\beta$ & 0.2291 & 0.0007 & $\beta$ & 0.5011 & 0.00320 & $\beta$ & 0.5022 & 0.0021 \\
\hline
\end{tabular}

Table 4: MLEs and SEs for faliure times data set

\begin{tabular}{|c|c|c|c|c|c|}
\hline \multirow{2}{*}{$\frac{\text { Model }}{\operatorname{BHEW}(\theta, \alpha, \beta)}$} & & & \multicolumn{3}{|l|}{ Estimates } \\
\hline & $\begin{array}{l}814.32 \\
(582.25)\end{array}$ & $\begin{array}{l}20.146 \\
(1.683)\end{array}$ & $\begin{array}{l}0.223 \\
(0.03)\end{array}$ & & \\
\hline PTL-W $(\lambda, \alpha, b)$ & $\begin{array}{l}-5.782 \\
(1.395)\end{array}$ & $\begin{array}{l}4.22865 \\
(1.167)\end{array}$ & $\begin{array}{l}0.658 \\
(0.039)\end{array}$ & & \\
\hline $\operatorname{MOEW}(\Upsilon, \beta, \alpha)$ & $\begin{array}{l}488.9 \\
(189.36)\end{array}$ & $\begin{array}{l}0.28 \\
(0.013)\end{array}$ & $\begin{array}{l}1261.96 \\
(351.1)\end{array}$ & & \\
\hline $\mathrm{GaW}(\alpha, \beta, \Upsilon)$ & $\begin{array}{l}2.377 \\
(0.378)\end{array}$ & $\begin{array}{l}0.8481 \\
(0.0005)\end{array}$ & $\begin{array}{l}3.53 \\
(0.665)\end{array}$ & & \\
\hline $\mathbf{D E W}(\theta, \lambda, \alpha, \beta)$ & $\begin{array}{l}0.4605 \\
(0.1531)\end{array}$ & $\begin{array}{l}26.343 \\
(0.000)\end{array}$ & $\begin{array}{l}1.731 \\
(0.059)\end{array}$ & $\begin{array}{l}0.0816 \\
(0.000)\end{array}$ & \\
\hline $\mathrm{KwW}(\alpha, \beta, \mathrm{a}, \mathrm{b})$ & $\begin{array}{l}14.433 \\
(27.09)\end{array}$ & $\begin{array}{l}0.2041 \\
(0.042)\end{array}$ & $\begin{array}{l}34.66 \\
(17.53)\end{array}$ & $\begin{array}{l}81.85 \\
(52.01)\end{array}$ & \\
\hline $\operatorname{WFr}(\alpha, \beta, a ; b)$ & $\begin{array}{l}630.94 \\
(697.9)\end{array}$ & $\begin{array}{l}0.30 \\
(0.03)\end{array}$ & $\begin{array}{l}416.1 \\
(232.36)\end{array}$ & $\begin{array}{l}1.17 \\
(0.36)\end{array}$ & \\
\hline $\mathrm{BW}(\alpha, \beta, \mathrm{a} ; \mathrm{b})$ & $\begin{array}{l}1.36 \\
(1.002)\end{array}$ & $\begin{array}{l}0.2981 \\
(0.06)\end{array}$ & $\begin{array}{l}34.2 \\
(14.8)\end{array}$ & $\begin{array}{l}11.5 \\
(6.7)\end{array}$ & \\
\hline $\operatorname{TMW}(\alpha, \beta, \Upsilon, \lambda)$ & $\begin{array}{l}0.2722 \\
(0.014)\end{array}$ & $\begin{array}{l}1 \\
\left(5.2 \times 10^{-5}\right)\end{array}$ & $\begin{array}{l}4.6 \times 10^{-6} \\
\left(1.9 \times 10^{-4}\right)\end{array}$ & $\begin{array}{l}0.4685 \\
(0.165)\end{array}$ & \\
\hline $\operatorname{KwTW}(\alpha, \beta, \lambda \mathrm{a}, \mathrm{b})$ & $\begin{array}{l}27.79 \\
(33.4)\end{array}$ & $\begin{array}{l}0.178 \\
(0.017)\end{array}$ & $\begin{array}{l}0.445 \\
(0.609)\end{array}$ & $\begin{array}{l}29.5 \\
(9.8)\end{array}$ & $\begin{array}{l}168.1 \\
(129.2)\end{array}$ \\
\hline M-BW $(\alpha, \beta, a, b, c)$ & $\begin{array}{l}10.15 \\
(18.7)\end{array}$ & $\begin{array}{l}0.163 \\
(0.02)\end{array}$ & $\begin{array}{l}57.417 \\
(14.063)\end{array}$ & $\begin{array}{l}19.39 \\
(10.02)\end{array}$ & $\begin{array}{l}2.004 \\
(0.66)\end{array}$ \\
\hline M-cW $(\alpha, \beta, \mathrm{a}, \mathrm{b}, \mathrm{c})$ & $\begin{array}{l}1.94 \\
(1.011)\end{array}$ & $\begin{array}{l}0.31 \\
(0.045)\end{array}$ & $\begin{array}{l}17.69 \\
(6.222)\end{array}$ & $\begin{array}{l}33.6388 \\
(19.994)\end{array}$ & $\begin{array}{l}16.7211 \\
(9.722)\end{array}$ \\
\hline TExGW $(\alpha, \beta, \lambda \mathrm{a}, b)$ & $\begin{array}{l}4.257 \\
(33.4)\end{array}$ & $\begin{array}{l}0.1532 \\
(0.017)\end{array}$ & $\begin{array}{l}0.098 \\
(0.609)\end{array}$ & $\begin{array}{l}5.23 \\
(0.76)\end{array}$ & $\begin{array}{l}1173.328 \\
(9.792)\end{array}$ \\
\hline
\end{tabular}


Mona Mustafa Elbiely / Journal of Mathematics and Statistics 2019, Volume 15: 122.135 DOI: $10.3844 / j m s s p .2019 .122 .135$

Table 5: $\mathrm{W}^{\star}$ and $\mathrm{A}^{\star}$ for faliure times data set

\begin{tabular}{lll}
\hline Model & $\mathrm{W}^{\star}$ & $\mathrm{A}^{\star}$ \\
\hline DEW & $\mathbf{0 . 0 9 0 1}$ & $\mathbf{0 . 6 4 7 1}$ \\
BHEW & 0.0917 & 0.8740 \\
PTLW & 0.1397 & 1.19390 \\
MOEW & 0.3995 & 4.44770 \\
GaW & 0.2553 & 1.94890 \\
KWW & 0.1852 & 1.50590 \\
WFr & 0.2537 & 1.95740 \\
BW & 0.4652 & 3.21970 \\
TMW & 0.8065 & 1.2050 \\
KWTW & 0.1640 & 1.36320 \\
MBW & 0.4717 & 3.26560 \\
McW & 0.1986 & 1.59060 \\
TExGW & 1.0079 & 6.23320 \\
\hline
\end{tabular}
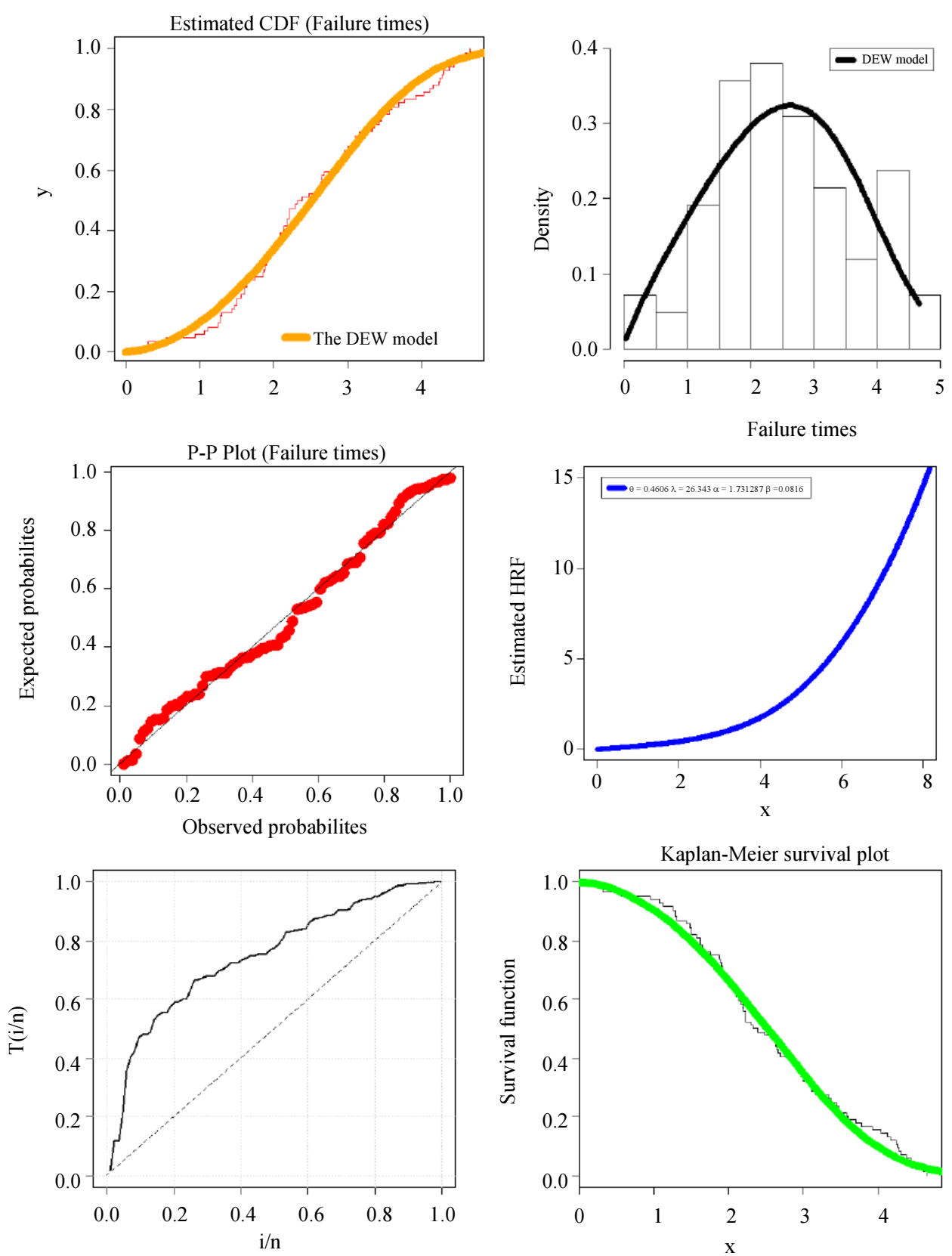

Fig. 3: CDF, PDF, P-P plot, HRF, TTT plot and Kaplan-Meier survival plot for data set I 

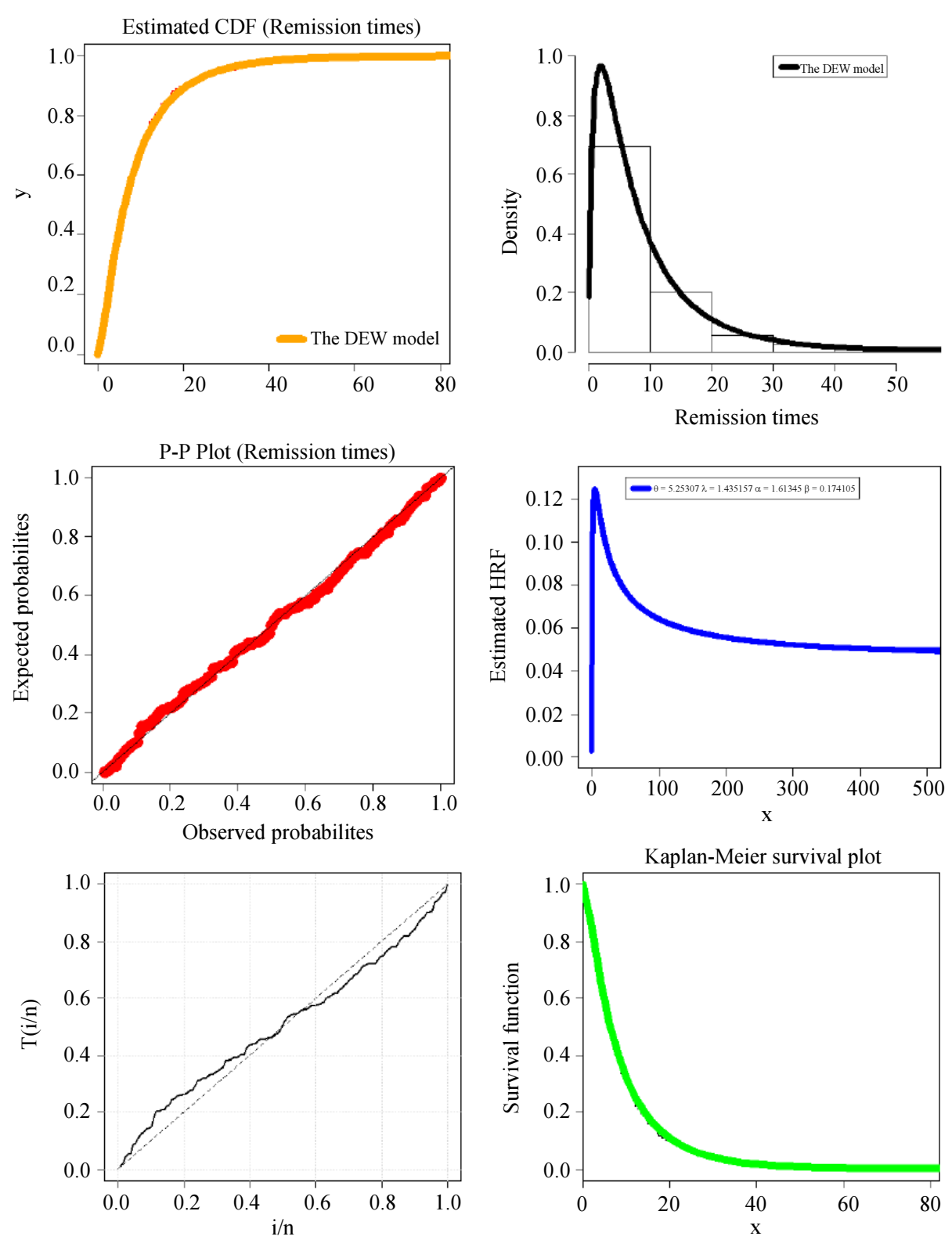

Fig. 4: CDF, PDF, P-P plot, HRF, TTT plot and Kaplan-Meier survival plot for data set II
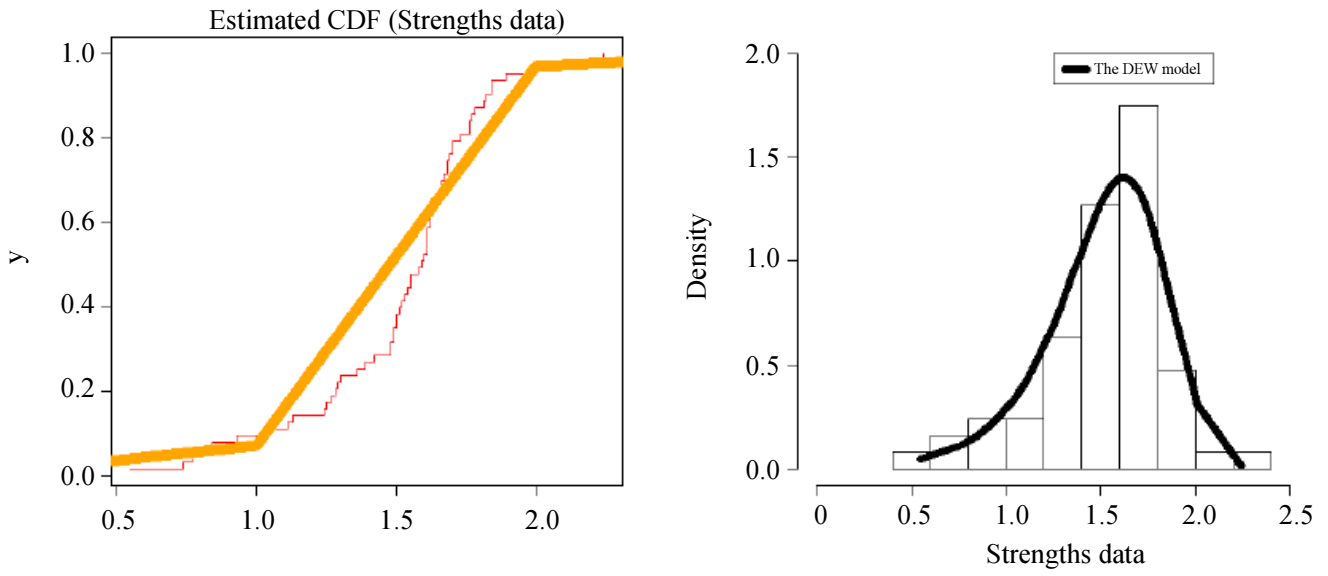

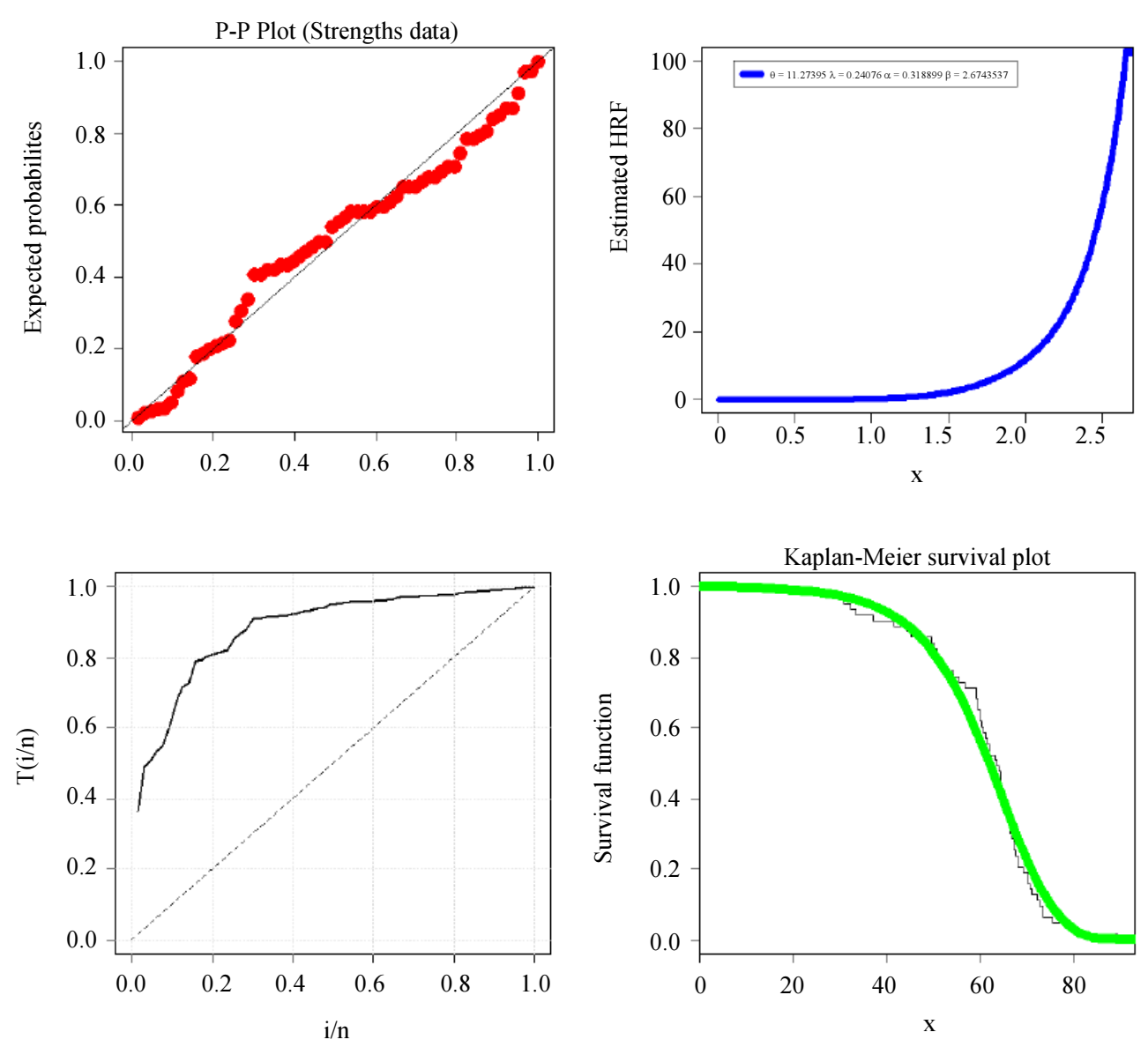

Fig. 5: CDF, PDF, P-P plot, HRF, TTT plot and Kaplan-Meier survival plot for data set III

\section{Modeling Remission Times}

The second data set denotes the remission times (in months) of a random sample of 128 bladder cancer patients. We intend to compare the fits of the DEW distribution with those of other models, namely: the WW, the Odd WW (OWW), the Gamma Exponentiated Exponential (GaEE) distributions, whose PDFs for $x>0$. Table 6 list the results of MLEs and SE for all fitted models. It is clear from Table 7 that the DEW distribution provides the best fit to remission times data set.

\section{Modeling the Strengths}

This data consists of 63 observations of the strengths of $1.5 \mathrm{~cm}$ glass fibers, originally obtained by workers at the UK National Physical Laboratory. For this data set, we compare the fits of the DEW distribution with some competitive models like BHEW, EW, TW and OLLW. Table 8 list the values of MLEs and SE for all models. This data have also been analyzed in a paper.
Table 6: MLEs and SEs for remission times data set

\begin{tabular}{lllll}
\hline Model & & & Estimates \\
\hline $\operatorname{WW}(\theta, \alpha, \beta)$ & 2.6594 & 0.6933 & 0.0270 & \\
& $(0.7129)$ & $(0.1707)$ & $(0.019)$ & \\
$\operatorname{OWW}(\theta, \alpha, \beta)$ & 11.158 & 0.0881 & 0.457 & \\
& $(4.545)$ & $(0.036)$ & $(0.077)$ & \\
$\operatorname{GaEE}(\theta, \alpha, \beta)$ & 2.114 & 2.601 & 0.008 & \\
& $(1.33)$ & $(0.56)$ & $(0.005)$ & \\
$\operatorname{DEW}(\theta, \lambda, \alpha, \beta)$ & 5.2531 & 1.4352 & 1.6135 & 0.174 \\
& $(22.47)$ & $(7.586)$ & $(2.369)$ & $(0.61)$ \\
\hline
\end{tabular}

Table 7: $\mathrm{W}^{\star}$ and $\mathrm{A}^{\star}$ for remission times data set

\begin{tabular}{lcc}
\hline Model & $\mathrm{W}^{\star}$ & $\mathrm{A}^{\star}$ \\
\hline DEW & 0.0462 & 0.3074 \\
WW & 0.1427 & 0.7811 \\
OWW & 0.4494 & 2.4764 \\
GaEE & 0.3150 & 1.7208 \\
\hline
\end{tabular}

Table 8: MLEs and SEs for the strengths data set

\begin{tabular}{lllll}
\hline Model & \multicolumn{3}{l}{ Estimates } \\
BHEW $(\theta, \alpha, \beta)$ & 908.64 & 21.127 & 0.5 \\
& $(577.31)$ & $(1.61)$ & $(0.06)$ & \\
EW $(\theta, \alpha)$, & 0.671 & 7.285, & 1.718 & \\
& $(0.249)$ & $(1.707)$ & $(0.09)$ & \\
TW $(\theta, \alpha, \beta)$ & -0.501 & 5.15, & 0.646 & \\
& $(0.27)$ & $(0.67)$ & $(0.024)$ & \\
OLLW $(\theta, \alpha, \beta)$ & 0.9439 & 6.0256 & 0.6159 & \\
& $(0.2689)$ & $(1.348)$ & $(0.016)$ & \\
DEW $(\theta, \lambda, \alpha, \beta)$ & 11.27 & 0.241 & 0.32 & 2.6 \\
& $(13.8)$ & $(0.11)$ & $(1.047)$ & $(0.4)$ \\
\hline
\end{tabular}


Table 9: $\mathrm{W}^{\star}$ and A for the strengths data set

\begin{tabular}{lll}
\hline Model & $\mathrm{W}^{\star}$ & $\mathrm{A}^{\star}$ \\
\hline DEW & 0.141 & 0.082 \\
BHEW & 0.316 & 1.73 \\
EW & 0.636 & 3.484 \\
TW & 1.036 & 0.169 \\
OLLW & 1.236 & 0.219 \\
\hline
\end{tabular}

\section{Concluding Remarks}

A new lifetime Exponentiated weibull model called the Dual Exponentiated Weibull Model (DEW) with various shapes of the HRF for modeling unimodal and bimodal data sets is introduced and studied along with its statistical properties. Before using the maximum likelihood method for estimating the unknown model parameters, we assessed its performance via a simulation study. The flexibility of the new model is illustrated via plots of the probability and hazard rate functions and three real data applications. The DEW model is very attractive to define special models with different types of hazard rates and its density can be symmetric or rightskewed or left-skewed or reversed $\mathbf{J}$ shape or unimodal or bimodal. The HRF of the DEW model can be increasing or increasing constant or decreasing or upside-down or bathtub or upside-down bathtub or constant or $\mathbf{J}$ shaped. The DEW model provides the best fit in modeling different types of data sets as illustrated using three real data sets.

\section{Acknowledgement}

The author gratefully acknowledges with thanks the very thoughtful and constructive suggestions and comments of the Editor-in-Chief and the reviewers which resulted in much improved paper.

\section{Author Contributions}

The author wrote and developed the paper.

\section{Ethics}

The author declares that there is no conflict of interests regarding the publication of this article.

\section{References}

Alizadeh, M., I. Ghosh, H.M. Yousof, M. Rasekhi and G.G. Hamedani, 2017. The generalized odd generalized exponential family of distributions: properties, characterizations and applications. J. Data Sci., 15: 443-466.

Bourguignon, M., R.B. Silva and G.M. Cordeiro, 2014. The Weibull-G family of probability distributions. J. Data Sci., 12: 53-68.
Brito, E., G.M. Cordeiro, H.M. Yousof, M. Alizadeh and G.O. Silva, 2017. Topp-leone odd log-logistic family of distributions. J. Stat. Computat. Simulat., 87: 3040-3058.

Cordeiro, G.M., A.Z. Afify, H.M. Yousof, R.R. Pescim and G.R. Aryal, 2017. The exponentiated Weibull-H family of distributions: Theory and applications. Mediterranean J. Mat., 14: 1-22.

Cordeiro, G.M., H.M. Yousof, T.G. Ramires and E.M.M. Ortega, 2018. The burr XII system of densities: Properties, regression model and applications. J. Stat. Computat. Simulat., 88: 432-456.

Gupta, R.D. and D. Kundu, 1999. Generalized exponential distributions. Australian New Zealand J. Stat., 41: 173-188.

Hamedani G.G., A.E, Korkmaz, M.C. Yousof and N.S. Butt, 2018. A new extended $G$ family of continuous distributions with mathematical properties, characterizations and regression modeling. Pak. J. Stat. Oper. Res., 14: 737-758.

Hamedani, G.G. H.M. Yousof, M. Rasekhi, M. Alizadeh and S.M. Najibi, 2017. Type I general exponential class of distributions. Pak. J. Stat. Oper. Res., 14: 39-55.

Hassan, A. S. and M. Elgarhy, 2016. A new family of exponentiated Weibull-generated distributions. Int. J. Math. Appli., 4: 135-148.

Khalil, M.G., G.G. Hamedani and H.M. Yousof, 2019. The burr $\mathrm{X}$ exponentiated Weibull model: Characterizations, mathematical properties and applications to failure and survival times data. Pak. J. Stat. Oper. Res., 20: 141-160.

Korkmaz, M.C., E. Altun, H.M. Yousof and G.G. Hamedani, 2019. The odd power lindley generator of probability distributions: properties, characterizations and regression modeling. Int. J. Stat. Probability, 8: 70-89.

Lee, E.T. and J.W. Wang, 2003. Statistical Methods for Survival Data Analysis. 1st Edn., Wiley, New York.

Mahmoudi, E., R.S. Meshkat, B. Kargar and D. Kundu, 2018. The extended exponenti-ated Weibull distribution and its applications. Stat. Anno, 4: 363-396.

Merovci, F., M. Alizadeh, H.M. Yousof and G.G. Hamedani, 2017. The exponentiated transmuted-G family of distributions: Theory and applications. Communicat. Stat. Theory Methods, 46: 10800-10822.

Mudholkar, G.S. and D.K. Srivastava, 1993. Exponentiated Weibull family for analyzing bathtub failure-real data. IEEE Trans. Reliability, 42: 299.302.

Nadarajah, S., G.M. Cordeiro and E.M.M. Ortega, 2013. The exponentiated Weibull distribution: A survey. Stat. Papers, 54: 839-877. 
Smith, R.L. and J.C. Naylor, 1987. A comparison of maximum likelihood and Bayesian estimators for the three-parameter Weibull distribution. Appl. Statist., 36: 358-369.

Weibull, W., 1951. A statistical distribution function of wide applicability. J. Appl. Mech. Trans., 18: 293-297.

Yousof, H.M., A.Z. Afify, G.G. Hamedani and G. Aryal, 2017a. The burr $X$ generator of distributions for lifetime data. J. Stat. Theory Applic., 16: 288.305.
Yousof, H.M., M. Alizadeh, S.M.A. Jahanshahiand, T.G. Ramires and I. Ghosh et al., 2017b. The transmuted topp-leone $\mathrm{G}$ family of distributions: Theory, char-acterizations and applications. J. Data Sci., 15: 723-740.

Yousof, H.M., M. Majumder, S.M.A. Jahanshahi, M.M. Ali and G.G. Hamedani, 2018. A new Weibull class of distributions: Theory, characterizations and applications, J. Stat. Res. Iran, 15: 45.83.

\section{Appendix A:}

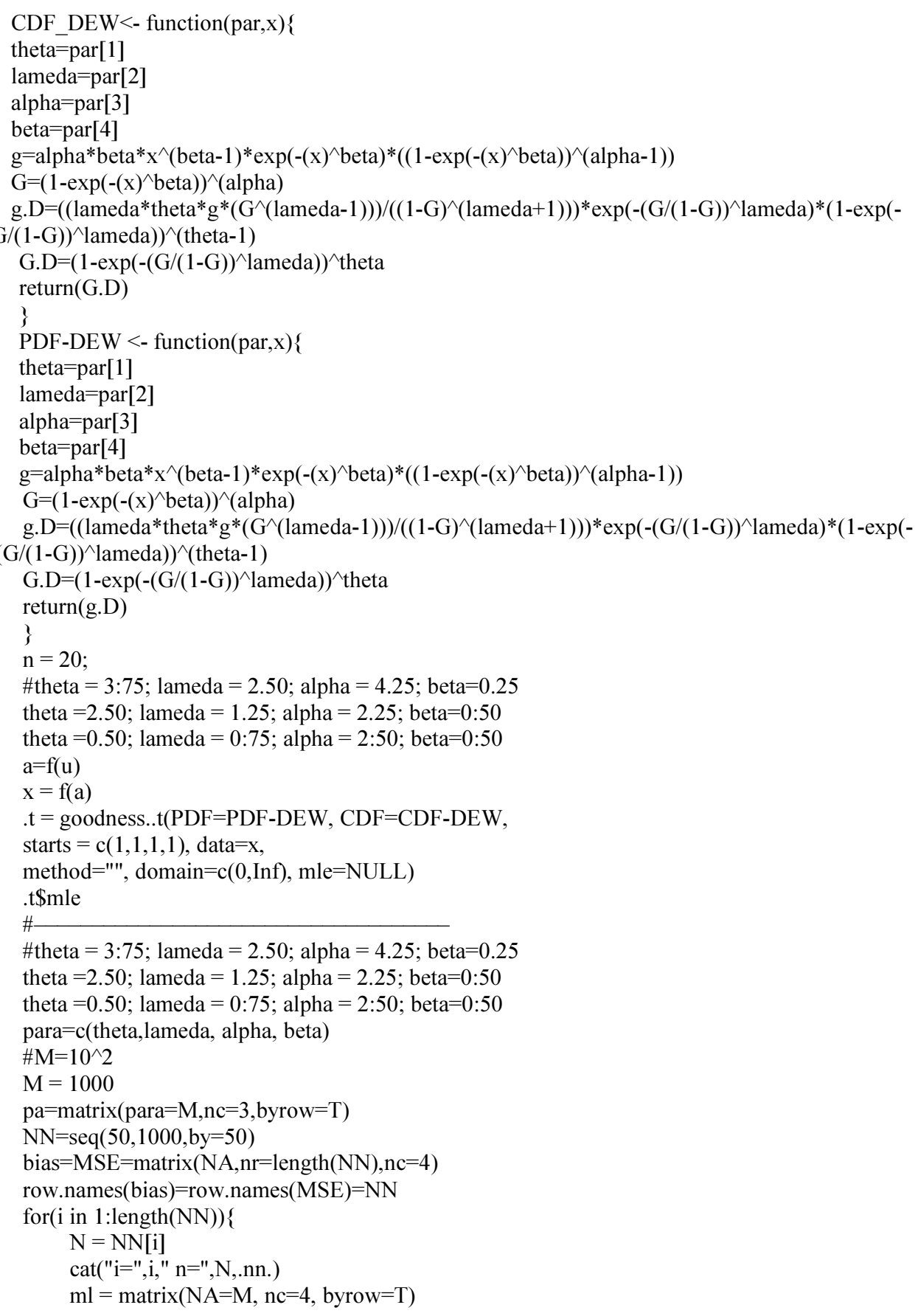




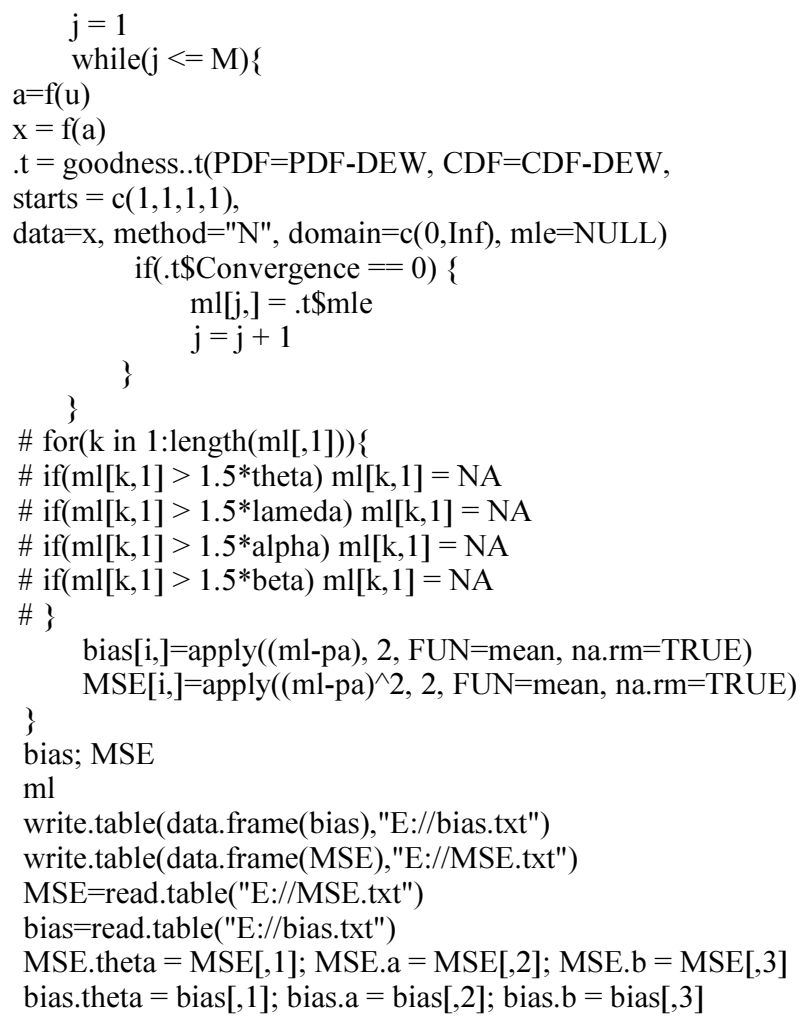

\section{Appendix B:}

Application I, II and III $\mathrm{x}=($ Data $)$

hist(x)

\#==

CDF_DEW <- function(, $)\{$

theta $=$ par[1]

lameda=par[2]

alpha $=$ par[3]

beta=par[4]

$\mathrm{g}=$ alpha*beta* $\mathrm{x}^{\wedge}(\text { beta-1 })^{*} \exp \left(-(\mathrm{x})^{\wedge}\right.$ beta $) *\left(\left(1-\exp \left(-(\mathrm{x})^{\wedge} \text { beta }\right)\right)^{\wedge}(\right.$ alpha-1 $\left.)\right)$

$\mathrm{G}=\left(1-\exp \left(-(\mathrm{x})^{\wedge} \text { beta }\right)\right)^{\wedge}($ alpha $)$

g.D $=\left(\left(\text { lameda*theta*g* }{ }^{*} \mathrm{G}^{\wedge}(\text { lameda-1) })\right) /\left((1-\mathrm{G})^{\wedge}(\text { lameda }+1)\right)\right)^{*} \exp \left(-(\mathrm{G} /(1-\mathrm{G}))^{\wedge}\right.$ lameda $) *(1-\exp (-$

$(\mathrm{G} /(1-\mathrm{G}))^{\wedge}$ lameda $\left.)\right)^{\wedge}($ theta-1)

$\mathrm{G} . \mathrm{D}=\left(1-\exp \left(-(\mathrm{G} /(1-\mathrm{G}))^{\wedge} \text { lameda }\right)\right)^{\wedge}$ theta

return(G.D)

\}

PDF DEW <- function(par,x)

theta= $\operatorname{par}[1]$

lameda $=$ par[2]

alpha $=$ par[3]

beta $=$ par[4]

$\mathrm{g}=$ alpha*beta* $\mathrm{x}^{\wedge}(\text { beta- } 1)^{*} \exp \left(-(\mathrm{x})^{\wedge}\right.$ beta $) *\left(\left(1-\exp \left(-(\mathrm{x})^{\wedge} \text { beta }\right)\right)^{\wedge}(\right.$ alpha-1 $\left.)\right)$

$\mathrm{G}=\left(1-\exp \left(-(\mathrm{x})^{\wedge} \text { beta }\right)\right)^{\wedge}($ alpha $)$

g. $D=\left(\left(\right.\right.$ lameda*theta*g* $\left(\mathrm{G}^{\wedge}(\right.$ lameda-1) $\left.)\right) /\left((1-\mathrm{G})^{\wedge}(\right.$ lameda +1$\left.\left.)\right)\right) * \exp \left(-(\mathrm{G} /(1-\mathrm{G}))^{\wedge}\right.$ lameda $) *(1-\exp (-$

$(\mathrm{G} /(1-\mathrm{G}))^{\wedge}$ lameda $\left.)\right)^{\wedge}($ theta-1)

$\mathrm{G} . \mathrm{D}=\left(1-\exp \left(-(\mathrm{G} /(1-\mathrm{G}))^{\wedge} \text { lameda }\right)\right)^{\wedge}$ theta

return(g.D)

\}

goodness.t(PDF=PDF-DEW, $\mathrm{CDF}=\mathrm{CDF}-\mathrm{DEW}$, starts $=\mathrm{c}(1,1,1,1)$, data $=\mathrm{x}$, method $="$ ", domain $=\mathrm{c}(0$, Inf $)$, mle $=$ NULL $)$ 